Jochen Bauer*, Thomas Wittenberg, Klemens Waldhör, Michael Hechtel, Martin Holzwarth, Julian Sessner, Bruno Ristok, Florian Böhle, Annamaria Schena, Annarita Falanga, and Jörg Franke

\title{
A Concept for Context Awareness in Smart Environments
}

https://doi.org/10.1515/cdbme-2020-3098

\begin{abstract}
All aspects of daily life increasingly include digitization. So-called ,smart home“ technologies, as well as ,wearables", are gaining attention from more and more dwellers. Therefore, sensor-based, individualized, AI-based services for improved post-intervention monitoring and therapy accompaniment will become feasible and possible if these systems offer a related context-awareness. This paper provides an approach on how to sense and interpret specific contexts with the help of wearables, smartwatches, smart home sensors, and emotion detection software.
\end{abstract}

Keywords: emotion recognition, smart home, smart living, smartwatches, wearables

\section{Motivation}

The internet of things (IoT) is currently growing, and related devices enter our daily life [1]. In the past 15 years, there has been an increasing amount of activities within the field of ambient assisted living (AAL) in order to support, increase and maintain the health of residents at home, hospitals, or nursing facilities. Nevertheless, the definition of AAL has changed over time [2]. Recent AAL definitions include more and more technical support systems, for example, wearables. Wearables represent IT systems, which reside on or inside the human body and include several sensors, which are most often combined with an IT-backbone application. Such wearables (as e.g. smartwatches, smartphones, smart-shoes, smart-textiles or

\footnotetext{
*Corresponding author: Jochen Bauer, Michael Hechtel, Martin Holzwarth, Julian Sessner, Jörg Franke, Institute for Factory Automation and Production Systems, Fürther Straße 246b, Nuremberg, Germany, e-mail: jochen.bauer@faps.fau.de Thomas Wittenberg, Fraunhofer Institute for Integrated Circuits IIS, Am Wolfsmantel 33, Erlangen, Germany

Klemens Waldhör, FOM University of Applied Sciences for Economics and Management, Zeltnerstraße 19, Nuremberg, Germany

Annamaria Schena, Annarita Falanga, Villa delle Ginestre srl, Via Torino 47 - 49, Volla, Italy

Bruno Ristok, C\&S Computer und Software GmbH in Augsburg, Wolfsgäßchen 1, Augsburg, Germany

Florian Böhle, easierLife GmbH Herrenstraße 50a, Karlsruhe, Germany
}

similar devices) are able to collect data in the surrounding of the person under surveillance and can carry out evaluations directly at the point of care, whose results are relevant for the dwellers [3]. Users' benefits will increase, if several complementary smart-living-related domains are combined into a single platform, as, e.g., for health, energy management or home automation domain [4]. Furthermore, in order to increase the value proposition as well as the acceptance of smart-home products for dwellers, it is useful to enable the usage of different communication protocols by offering an independent middleware layer like openHAB [5]. Smartwatches are an ideal option to gather data in and outside of the home environment, for example, to detect the wearer's position or dangerous situations [6, 7]. Additionally, smartwatches are useful to interact with human-machine interface (HMI) channels, for example, sound- or screen-based interfaces. Furthermore, service robots are currently gaining more and more attention and enter the consumer market. The spectrum of service robots ranges from established vacuum cleaner and lawnmower robots to communication robots like Pepper, Sanbot, or Temi $[8,9,11]$. Such robots are capable of creating maps of their environment or are offering camera-based object identification scenarios to detect possible tumbles or falls [11] and potentially endangering obstacles. Moreover, object identification applications can be used to determine the contents of the fridge or the storage room or to identify and recognize known people in a household. It is predictable that shortly such robot platforms will include additional sensors to enrich data acquisition and analysis possibilities.

Besides the amount of available data inside the local network, there is another enabler for improving context awareness: Artificial intelligence (AI). Among other things, AI has recently been established for various vital applications such as natural language processing (including speech recognition and speech-to-command applications) and image-based object identification. Additionally, several research activities are currently applying AI methods for process optimization in the smart living domain [13]. These strategies make it possible to substitute sensors and detect the user's needs more robust due to cloud-based data analysis. Devices or groups of devices, which can offer such AI-driven behavior are called thinking objects (TO) [13]. Nevertheless, to achieve such 
high-end functionality soon, seamless integration of cloudbased AI-services, a middleware interoperability layer and IoT-supported devices will be necessary.

Due to aggregated information, it is possible to create individually shaped use cases. To accomplish this goal, contextawareness is mandatory [12]. For example, for chronic diseases such as obesity treatment, therapists consider individually strategies to support patients' efforts to change their behavior, i.e. by increasing their daily activity, improve their food selection, and healthy habits in general [14? ? ]. Another use case that will profit from context-awareness is fall detection because the appropriate chain-of-reaction strongly depends on the current context [15] of the individual. Moreover, existing algorithms for detecting, analyzing, and differentiating the activities of daily living (ADL) and events of daily living (EDL) will profit from an improved context-awareness as well.

In section 2, we will identify current challenges, which need to be addressed to enable robust context-awareness in smart living environments for specific use cases. Afterwards, in section 3, we provide a concept for an approach with respect to the mentioned challenges, which will be discussed at the end of the paper.

\section{Challenges}

As mentioned above, there exist lots of new possibilities for detecting users' environmental context and preferred activities. Improved context-awareness will enrich many smart-living-, home-care- and nursing-environment-related use cases. Nevertheless, these opportunities will lead to new challenges: smart devices need to send, receive, and analyze adequate and sufficient data from different subsystems with possibly different protocols and offered by several vendors. Therefore, novel data sovereignty concepts need to consider such multi-infrastructure systems. To fulfill data sovereignty, these concepts need to consider the complete lifecycle of each data entry, thus the user needs appropriate mechanisms to configure and manage third-party-access to his data. Summing up, innovative and robust identity and access management (IAM) methods need to be implemented.

Smart environments can help to identify users and the corresponding user's position in the house. Several methods exist for identification and positioning challenges. All of these methods have advantages and disadvantages. Common technologies to locate and identify users are camera- or devicebased. For positioning Bluetooth- and other network-related methods are established, which are often using signal strengths to determine a user's position in an environment [10]. A useful precision for smart home applications is to detect the user's position and the current room. After this type of information is available for the system, it is possible to gather relevant data within this room and deliver user-specific output messages at the best possible location and on the most preferred type of device for the tenant.

Wearable devices like smartwatches are an easy way to identify an individual because this information is usually already stored on the device by default. With their ability to recognize ADLs and EDLs, as well as health-related data such as pulse, ECG, or physical activity, smartwatches (and similar devices) may support elderly people to remain within their private homes and not move into retirement or nursing homes. Nevertheless, smartwatches come with some challenges, especially how, in case of an automatically detected health hazard, adequate help can be brought to the patient. One key challenge is to find the context in which the need for support occurs. Smartwatches are useful because they can be worn everywhere - within the house and outside. If the accident occurs outside of the residence, the exact location can be determined relatively easily by using GPS. Inside buildings, this task is more difficult, relying only on smartwatches, as it requires further location information provided by the services of a smart home, for example WiFi-based signal strength values. Context-awareness is based on tuples of attributes and corresponding values, for example, „user“, „location of user“, „speed of user“, ,time of day“ or „detected activities“. Hence, the first challenge is to avoid false-negative warnings (e.g., proposing a tumble, although no tumble occurred) as this will lead to negative consequences if external support is called and it was not necessary. The second challenge is to avoid alarms in situations where an alarm is detected correctly, but the location and time are inappropriate for an output of the warning, and the alarm can be postponed. An example for this is activating a notice to remind a user to consume some water or take some medications, e.g. while driving or when being involved in some public activity, for example during a conversation. Thus, smartwatches and related systems need to learn and adopt when it will be wise to skip or postpone an alert, a warning, or a notice.

Emotions can be classified dimensionally or categorically. According to Wundt [16], the dimensions include pleasurable or unpleasurable, arousing or subduing and strain or relaxation. Alternatively, the categorical emotion classification introduced by Plutchik [17] can be divided into joy, trust, fear, surprise, sadness, disgust, anger and anticipation. In 1978 Ekman and Friesen [18] developed the Facial Action Coding System (FACS) to describe facial expressions. Based on FACS Action Units (AU) can be described and recognized to implement an emotional state detection (dimensional and categorical). With the FACS system, 46 AUs were defined, such as ,twisting the nose“ or ,rising the left eyebrow“İn addition, 
for each AU its strength of expression is coded from „A " to „E“ with „E“ being the maximum. These AUs can be associated with the proficiency level and assigned to basic emotions. In this way, it is possible to assign basic emotions, an important characteristic for context-awareness, from which recommendations can be critically examined. For example, the „SHORE“ software can be used to detect the basic emotions „,happy“, „sad“, „,surprised“ and „,angry“. This kind of automatic differentiation is advantageous, but within automated of context recognition, the recognition and differentiation of the AUs must be accelerated and refined.

To increase users' value proposition, it is important to offer interoperable system architecture approaches, like the Reference Architecture Modell for Industry 4.0 [19] and adapt that to the smart living domain. Currently, more and more middleware systems are enabling interoperability by adding device abstraction layers as well as semantic information. In addition to that, concepts arise to strive for interoperability on the business model layer as well: service providers are able to configure their business model and the system will make sure that the companies can access devices' functions successfully. A high interoperability level is beneficial to context-awareness in two ways: first, sensors and middleware can enrich data for the AI services by adding semantic information. These AI services can then verify the assumed context or can detect the context themselves and send it back to the smart home devices or middleware.

\section{Approach}

Concerning the aforementioned challenges, our approach focuses on enabling context-awareness specifically for the smart living domain. The described concept will be integrated into the ForeSight platform approach - an AI-driven platform for context-aware smart living services [13]. ForeSight offers the so-called TOs to service providers and dwellers. This approach will be demonstrated by describing two context-specific use case adaptions: First, how the context-awareness of existing activity and nutrition recommender systems [14] ,smart kitchen" can be improved. The second use case is the contextaware fall detection with a flexible chain of reaction scheme. Common AI-driven services, that are relevant for lots of use cases, are bundled and accessible as so-called ForeSight base services, for example the services „Object Identification“ and „Activity Detection“. Moreover, it will be possible to communicate between the system components bidirectionally and semantically enriched. Thus, it is possible that detected contexts can be transferred to other modules and verified. The following use case descriptions will show this in more detail.
The first use case is a context-aware smart kitchen scenario supporting the treatment of metabolic syndrome. Therefore, tenants need to raise their physical activity and improve their food choices, so to speak: ,live a healthier life“. Two recommender systems are available to achieve that goal, a nutrition recommender system (NRS) and an activity recommender system (ARS). ARS and NRS are parts of the the HealthRecapproach [14] and the health-related aspect is considered due to the NutriScale integration. Person A starts the day and wants to have breakfast. The system checks the fridge and storage room contents to figure out, if the meal can be prepared without further interruptions and the system recommends the most preferred meals and healthier alternatives. Simultaneously the service robot visits the tenant and detects the emotional state right after the recommendation was made. If it seems helpful, a dialog between the robot and the tenant will bei initialized to configure the meal recommendation appropriately. After the user selects the meal, household devices will be configured automatically. Third party services can offer additional context information like weather conditions or calendar data to evaluate how much time for the meal is actually available. After the meal, the ARS will recommend a physical exercise event, for example jogging. The robot takes the same role as before, checking the emotional state, when the recommendation has been offered. Smartwatch, fitness tracker or smart textiles will track the physical activity and will create relevant reports for the overall system.

The second use case is context-aware fall detection. The chain of reaction is adapted in relation to the detected context. Person B, 75 years old, is under surveillance regarding overall activity within and outside the house. Noticeable events will be detected and reported to the tenant. Several tools acquire this data, for example changed status of switches, energy consumption beacon-based positioning and smartwatches are gathering physical data. Existing algorithms are extended to determine current contexts to detect a fall and handle this event appropriately, for example initialize a dialogue or detect false alarms. If some intelligent floors are available, the system will consider the data due to available interfaces. After a fall has been detected and verified by tenant's smartwatch and service robot, possible options are identified, for example asking a neighbor for help in combination with an automatic unlocking of the door or calling a predefined contact person. Of course there will be an immediate emergency call, if that is necessary.

The project ERIK [9] currently researches the detection and differentiation of emotions to support robot based trainings of socio-emotional abilities for children with autism spectrum disorders. Therefore, the software is adapted and integrated into the humanoid robot Pepper using the robotic operating system ROS. In addition to Pepper, other cost-effective robot platforms, such as the Temi robot, which are currently 
available, will make it possible to cover other applications in business models in an economically viable manner. In the future, FACS-based approaches of emotion detection and interpretation need to be implemented and integrated into robot platforms (e.g. Pepper or Temi). Therefore, a robust and efficient implementation is crucial. Based on available ForeSight base services, the emotion detection might be integrated into the mentioned specific recommender systems for nutrition and activity. Moreover, it is appropriate that the robot will look for the tenant in need after a fall detection event has been detected. After arriving at the tenant the dialogue to find appropriate reactions will be with camera-based emotion detection in the robot. Identified contexts can be sent back to the smart home middleware system.

\section{Discussion}

In the next step, the described context-awareness concept needs to be verified through expert interviews. Afterwards, other context-aware use cases will be determined and the base services extended. At the end of 2022, these described contextaware use cases shall be available and evaluated in living labs and in Future Living Berlin, a real-world site for an AI-driven smart living environment.

Context-awareness enables the possibility to use singleperson households systems in multi-tenants households as well. There exists an enormous potential for the usage of service robots, which can add lots of relevant information to verify context-awareness and help as an HMI. The ForeSight architecture based on different modules and corresponding interfaces is able to integrate such service robots. With the combination of a smart home service platform and a smartwatch in combination with a service robot and only one bluetooth beacon per room, it seems likely to detect which user is in which room and one of the major challenges for modern smart living systems will be addressed by this concept.

\section{Author Statement}

Research funding: this work was supported by the BMWi (ForeSight) and BMBF (ERIK). Conflict of interest: Authors state no conflict of interest.

\section{References}

[1] Statista. Marktprognosen zum Internet der Dinge. Deutschland: 2017.

[2] Calvaresi, D. et al (2017) Exploring the ambient assisted living domain: a systematic review. J Ambient Intell Human Comput 8, 239-257
[3] Piwek L et al (2016) The Rise of Consumer Health Wearables: Promises and Barriers. PLoS Med 13(2): e1001953.

[4] Bauer, J.et al(2014) Die intelligente Wohnung als Baustein im Internet der Dinge: Potenzialanalyse und Konzept einer domänenübergreifenden Lösung. Proc's Erste Transdisziplinäre Konferenz zum Thema Technische Unterstützungssysteme, die die Menschen wirklich wollen (pp. 298-307).

[5] Parocha, R. \& Macabebe, E (2019) Implementation of Home Automation System Using OpenHAB Framework for Heterogeneous loT Devices Proc's International Conference on Internet of Things and Intelligence System (IoTaIS), pp. 67-73.

[6] Lutze R. \& Waldhör K. (2015) Smart Watches as Next Generation Home Emergency Systems Proc's 8th Congress of Active Assisted Living.

[7] Waldhör K. (2018) Anwendungen von Smartwatches und Wearables im Betrieblichen Gesundheitsmanagement. In: Matusiewicz D. et al. (eds) Digitales Betriebliches Gesundheitsmanagement. Springer Gabler, Wiesbaden

[8] Bauer, J. (2019) Anwendungsbeispiel: Servicerobotik im Haushalt. In: Müller, R. et al. Handbuch Mensch-RoboterKollaboration, Hanser, München.

[9] Simon M et al. (2019) Akzeptanzrisiken in der MenschRoboter-Interaktion - Nutzerzentrierte Erhebung von ethischen, rechtlichen und sozialen Implikationen (ELSI) am Beispiel einer Roboterplattform für Kinder mit eingeschränkten sozio-emotionalen Fähigkeiten. Proc's Mensch und Computer.

[10] Bauer, J., et al. (2018) SmartLocate. In: Uckelmann, D., (ed.) Proc's Smart Public Building, pp. 15-28.

[11] Bauer, J., et al. (2018) Camera-based fall detection system with the service robot sanbotELF. In: Uckelmann, D., (ed.) Proc's Smart Public Building. pp. 15-28

[12] Abowd, G., et al. (1999) Towards a Better Understanding of Context and Context-Awareness. Proc's 1st International Symposium on Handheld and Ubiquitous Computing, volume 1707, pages 304-307.

[13] Bauer J, et al. (2019) ForeSight - Platform Approach for Enabling Al-based Services for Smart Living. Proc's 17th ICOST.

[14] Bauer J. et al (2016) HealthRec - Recommender System to support the Treatment of metabolic Syndrom in a Smart Home. Proc's 9th Congress of Active Assisted Living.

[15] Pannurat, N., et al. (2014) Automatic Fall Monitoring: A Review. Sensors 2014, 14, 12900-12936.

[16] Wundt, W. (1874) Grundzüge der physiologischen Psychologie. Engelmann, Leipzig 1874, S. $800 f$.

[17] Plutchik, R. (1980) EMOTION: A Psychoevolutionary Synthesis. Harper \& Row.

[18] Ekman P \& Friesen W (1978) Facial Action Coding System: A Technique for the Measurement of Facial Movement. Con. Psychol., Palo Alto, Calif.

[19] VDI/VDE-GMA (2015) Zentralverband Elektrotechnik- und Elektronikindustrie e.V., Referenzarchitekturmodell Industrie 4.0 (RAMI 4.0) 